\title{
Summation of a Slowly Convergent Series Arising in Antenna Study
}

\author{
By Chi Fu Den
}

Abstract. An equivalent series for the slowly convergent series

$$
\sum_{n=1}^{\infty}\left[\int_{-\pi / 2}^{\pi / 2} \cos ^{\alpha} \theta \cos (n \epsilon \sin \theta)\right]^{2} / n
$$

which arises in antenna theory is obtained. The new form is found to consist of two rapidly convergent series for small $\epsilon$.

A recent study of the electromagnetic radiation from cylindrical structures [1], [3] requires the evaluation of a slowly convergent series $S_{1}=\pi^{2} \sum_{n=0}^{\infty}\left[J_{0}(n \epsilon)\right]^{2} / n$ where $J_{0}(n \epsilon)$ is the zeroth order Bessel function, and $\epsilon$ is a small positive constant. The expression above is a special case of the series

$$
S(\alpha)=\sum_{n=1}^{\infty}\left[p_{n}(\alpha)\right]^{2} / n
$$

where

$$
p_{n}(\alpha)=\int_{-\pi / 2}^{\pi / 2} \cos ^{\alpha} \theta \cos (n \epsilon \sin \theta) d \theta, \quad \alpha>-1 \text { and } 0<\epsilon .
$$

One notes that in the case $\alpha=0$

$$
p_{n}(0)=\int_{-\pi / 2}^{\pi / 2} \cos (n \epsilon \sin \theta) d \theta=\pi J_{0}(n \epsilon)
$$

and therefore $S(0)=S_{1}$. The aim of this brief is to obtain a more rapidly convergent series that is equivalent to Eq. (1).

Substituting Eq. (2) into Eq. (1) and interchanging the order of summation and integration results in

(3) $S(\alpha)=\int_{-\pi / 2}^{\pi / 2} \cos ^{\alpha} \theta\left\{\int_{-\pi / 2}^{\pi / 2} \cos ^{\alpha} \theta^{\prime}\left[\sum_{n=1}^{\infty} \cos (n \epsilon \sin \theta) \cos \left(n \epsilon \sin \theta^{\prime}\right) / n\right] d \theta^{\prime}\right\} d \theta$.

It is well known that

(4) $\sum_{n=1}^{\infty} \cos (n \epsilon \sin \theta) \cos \left(n \epsilon \sin \theta^{\prime}\right) / n=-\frac{1}{2} \ln 2\left|\cos (\epsilon \sin \theta)-\cos \left(\epsilon \sin \theta^{\prime}\right)\right|$.

Employing the Taylor expansion for cos $y$, the difference of two cosine functions in the vertical bars can be written as

$$
\cos (\epsilon \sin \theta)-\cos \left(\epsilon \sin \theta^{\prime}\right)=\frac{\epsilon^{2}}{4}\left(\cos 2 \theta-\cos 2 \theta^{\prime}\right)(1-A)
$$

Received June 24, 1968, revised December 13, 1968. 
where

(6) $A=\frac{2 \epsilon^{2}}{4 !}(x+y)-\frac{2 \epsilon^{4}}{6 !}\left(x^{2}+x y+y^{2}\right)+\frac{2 \epsilon^{6}}{8 !}\left(x^{3}+x^{2} y+x y^{2}+y^{3}\right)-\cdots$,

(7) $x=\sin ^{2} \theta$ and $y=\sin ^{2} \theta^{\prime}$.

Substitution of Eq. (5) into Eq. (4) leads to

$$
\begin{aligned}
& \sum_{n=1}^{\infty} \cos (n \epsilon \sin \theta) \cos \left(n \epsilon \sin \theta^{\prime}\right) / n \\
& =\ln \frac{2}{\epsilon}-\frac{1}{2} \ln 2\left|\cos 2 \theta-\cos 2 \theta^{\prime}\right|-\frac{1}{2} \ln |1-A| .
\end{aligned}
$$

If $|A|<1$ we can expand the last term in the Taylor series as follows

(9) $\quad \frac{1}{2} \ln |1-A|=\frac{1}{2} \ln (1-A)=-\frac{1}{2}\left(A+\frac{1}{2} A^{2}+\frac{1}{3} A^{3}+\cdots\right)$.

Inserting Eq. (9) in Eq. (8) and acknowledging that $A$ is defined as in Eq. (6) we obtain

$$
\begin{aligned}
& \sum_{n=1}^{\infty} \cos (n \epsilon \sin \theta) \cos \left(n \epsilon \sin \theta^{\prime}\right) / n \\
& \quad=\ln \frac{2}{\epsilon}+\sum_{n=1}^{\infty} \cos 2 n \theta \cos 2 n \theta^{\prime} / n+\frac{\epsilon^{2}}{24}(x+y) \\
& \quad+\frac{\epsilon^{4}}{2880}\left(x^{2}+6 x y+y^{2}\right)+\frac{\epsilon^{6}}{181440}\left(x^{3}+15 x^{2} y+15 x y^{2}+y^{3}\right)+\cdots .
\end{aligned}
$$

It is desired to determine the condition for which the inequality $|A|<1$ is satisfied. The exact form of that condition is not known. However, the upper bound of $A$ can be obtained readily. Since the absolute value of $\sin \theta$ is always less than or equal to unity, we see from Eq. (6)

$$
|A| \leqq \frac{4 \epsilon^{2}}{4 !}+\frac{6}{6 !} \epsilon^{4}+\frac{8}{8 !} \epsilon^{6}+\cdots=\frac{\sinh (\epsilon)}{\epsilon}-1 .
$$

Consequently, if the condition

$$
(\sinh (\epsilon) / \epsilon)-1<1
$$

or

$$
(\sinh (\epsilon) / \epsilon)<2
$$

is satisfied, then the inequality $|A|<1$ is always true. We acknowledge that (11) is more stringent than we really need.

Substituting Eq. (10) into Eq. (3) and interchanging the order of integration and summation for the series $\sum_{n=1}^{\infty} \cos 2 n \theta \cos 2 n \theta^{\prime} / n$ we arrive at

$$
\begin{aligned}
S(\alpha)= & C_{0}{ }^{2} \ln (2 / \epsilon)+f(\alpha)+\frac{\epsilon^{2}}{12} C_{0} C_{1}+\frac{\epsilon^{4}}{1440}\left(C_{0} C_{2}+3 C_{1}{ }^{2}\right) \\
& +\frac{\epsilon^{6}}{90720}\left(C_{0} C_{3}+15 C_{1} C_{2}\right)+\cdots,
\end{aligned}
$$


where

$$
\begin{aligned}
C_{n} & =\int_{-\pi / 2}^{\pi / 2} \cos ^{\alpha} \theta \sin ^{2 n} \theta d \theta, \quad n=0,1,2, \cdots \\
f(\alpha) & =\sum_{n=1}^{\infty}\left[\int_{-\pi / 2}^{\pi / 2} \cos ^{\alpha} \theta \cos 2 n \theta d \theta\right]^{2} / n .
\end{aligned}
$$

Making use of the following definite integrals [2]

$$
\int_{0}^{\pi / 2} \cos ^{\nu-1} z \sin ^{\mu-1} z d z=\frac{1}{2} \frac{\Gamma(\mu / 2) \Gamma(\nu / 2)}{\Gamma((\mu+\nu) / 2)}, \quad \operatorname{Re} \mu>0, \quad \operatorname{Re} \nu>0
$$

and the reflection formula for the Gamma function

$$
\Gamma(z) \Gamma(1-z)=\frac{\pi}{\sin \pi z}, \quad z \neq \text { integer },
$$

and Legendre's duplication formula, it can be shown that

$$
\begin{aligned}
& C_{0}=\sqrt{ } \pi[\Gamma((1+\alpha) / 2) / \Gamma(1+\alpha / 2)] \\
& C_{n}=\sqrt{ } \pi\left[\Gamma\left(\frac{1+\alpha}{2}\right) / \Gamma\left(1+\frac{\alpha}{2}\right)\right] \prod_{k=0}^{n-1} \frac{2 k+1}{2(n-k)+\alpha}, \quad n=1,2, \cdots .
\end{aligned}
$$

Also from [2] we find

$$
\begin{aligned}
& \int_{-\pi / 2}^{\pi / 2} \cos ^{\alpha} \theta \cos 2 n \theta d \theta \\
&=(-1)^{n+1} \frac{2}{\sqrt{ } \pi} \sin \left(\frac{\alpha \pi}{2}\right) \Gamma\left(\frac{1+\alpha}{2}\right) \Gamma\left(1-\frac{\alpha}{2}\right) \frac{d_{n}(\alpha)}{2 n+\alpha},
\end{aligned}
$$

where

$$
\begin{aligned}
& d_{1}(\alpha)=1 \\
& d_{n}(\alpha)=\prod_{k=1}^{n-1} \frac{2(n-k)-\alpha}{2(n-k)+\alpha}, \quad n \geqq 2,
\end{aligned}
$$

and $\alpha>-1$.

Substituting Eq. (18) into Eq. (14) results in

$$
f(\alpha)=\frac{4}{\pi}\left[\sin \frac{\alpha \pi}{2} \Gamma\left(\frac{1+\alpha}{2}\right) \Gamma\left(1-\frac{\alpha}{2}\right)\right]^{2} \sum_{n=1}^{\infty} \frac{\left[d_{n}(\alpha)\right]^{2}}{n(2 n+\alpha)^{2}} .
$$

Note that for $-1<\alpha<2, d_{n}(\alpha)$ is a monotonically decreasing function of both $n$ and $\alpha$, and, in particular,

$$
d_{n}(0)=1, \quad d_{n}(1)=\frac{1}{2 n-1} ;
$$

therefore Eq. (12) is seen to be represented by two rapidly convergent series for small $\epsilon$. 
The author is indebted to N. A. Strakhov and J. P. Cretella for their interest and help.

Bell Telephone Laboratories, Incorporated

Murray Hill, New Jersey 07974

1. C. F. Den, Admittance of a Wedge Excited Co-axial Antenna with a Plasma Sheath, Technical Report 5825-9-T, University of Michigan, Radiation Laboratory, Ann Arbor, Mich., 1966.

2. I. S. GRADŠTeǏn \& I. M. RYžIK, Table of Integrals, Series, and Products, Fizmatgiz, Moscow, 1963; English transl., Academic Press, New York, 1965, pp. 369, 372. MR 28 \#5198; MR 33 \#5952.

3. P. J. B. Clarricoats \& A. A. Oliner, "Improved theory of propagation through slotted circular waveguide," Electronics Letters, v. 3, 1967, pp. 279-282. 